\title{
An Epidemiologic Perspective on Scaphoid Fracture Treatment and Frequency of Nonunion Surgery in the USA
}

\author{
Christopher J. Dy, MD, MPH • Nikolas H. Kazmers, MD, MSE • Jack Baty, MS • Kerry Bommarito, PhD, MPH • \\ Daniel A. Osei, MD, MSc
}

Received: 6 February 2018/Accepted: 3 May 2018/Published online: 4 June 2018

(C) Hospital for Special Surgery 2018

\begin{abstract}
Background: Scaphoid fractures treated nonoperatively and operatively may be complicated by nonunion. Questions/Purposes: We sought to test the primary hypothesis that the incidence density of scaphoid fracture treatment is higher than previously estimated, to determine the frequency and risk factors for nonunion treatment, and to determine whether the frequency of surgical treatment increased over time. Methods: The MarketScan ${ }^{\circledR}$ database was queried for all records of treatment (casting and surgery) for closed scaphoid fractures over a 6-year period. We examined subsequent claims to determine frequency of additional procedures for nonunion treatment (revision fixation or vascularized grafting occurring 28 days or more after initial treatment). Trend analyses were used to determine whether changes in frequency of surgical treatment or revision procedure occurred. Results: The estimated incidence density of scaphoid fracture is 10.6 per 100,000 personyears in a commercially insured population of less than 65 years of age. Of 8923 closed scaphoid fractures, 29 and $71 \%$ were treated with surgery and casting, respectively. The frequency of surgical treatment rose significantly, from $22.1 \%$ in 2006 to $34.1 \%$ in 2012 . The frequency of
\end{abstract}

Electronic supplementary material The online version of this article (https://doi.org/10.1007/s11420-018-9619-3) contains supplementary material, which is available to authorized users.

C. J. Dy, MD, MPH · J. Baty, MS · K. Bommarito, PhD, MPH

Department of Orthopaedic Surgery, Washington University School of Medicine,

Saint Louis, MO, USA

N. H. Kazmers, MD, MSE $(\bowtie)$

Department of Orthopaedics, University of Utah,

Salt Lake City, UT, USA

e-mail: nkazmers@gmail.com

D. A. Osei, MD, MSc

Hospital for Special Surgery,

New York, NY, USA nonunion treatment was $10.8 \%$ after surgery and 3\% after casting; neither changed over time. Younger age, male sex, and surgical treatment are associated with a higher risk of nonunion treatment. Conclusions: Our estimated incidence of scaphoid fracture is higher than previously reported. The increased enthusiasm in the USA to surgically treat scaphoid fractures is reflected by our trend analysis. The frequency of surgical treatment for presumed nonunion after initial surgical management for closed scaphoid fractures exceeded $10 \%$. Given the increased utilization of surgery, surgeons and patients should be aware of the frequency of nonunion treatment to inform treatment decisions.

Keywords casting epidemiology fracture nonunion . scaphoid· surgery treatment

\section{Introduction}

The reported incidence density of scaphoid fractures in general populations varies widely, ranging from 1.47 per 100,000 person-years to 39 per 100,000 person-years [ 9 , $13,15,17,21]$. The diversity of these study populations from Sweden, Finland, and the USA likely contributes to this variation. Additionally, the difficulty in arriving at early, definitive diagnosis of scaphoid fracture likely contributes, as well; up to $30 \%$ of scaphoid fractures are missed on initial plain radiographs [1-3]. A more accurate reflection of the incidence of scaphoid fractures is necessary to better understand the related epidemiologic burden of these fractures in the USA. Also, a population-based assessment of the trends in scaphoid fracture treatment and rates of union would aid in understanding the relative value of each treatment option.

While retrospective case series and reports regarding nonunion and post-traumatic arthritis following scaphoid fractures abound in the literature, the relative frequency of nonunion is unknown. Determining the rate of nonunion is further complicated by the fact that fracture location, 
displacement, treatment type, and timing of treatment may all affect rates of union $[8,18,20]$. The purpose of this population-based study was to estimate the incidence density of scaphoid fracture treatment, the frequency of subsequent treatment for nonunion, and risk factors for nonunion treatment in the USA. The secondary aim was to analyze trends in the treatment of scaphoid fractures and the frequency of scaphoid nonunion treatment from 2006 to 2012. Our primary hypothesis was that the incidence density of scaphoid fractures would be higher than previously estimated. We examined our secondary hypothesis that the frequency of surgical treatment would increase over time using trend analysis.

\section{Materials and Methods}

We used the Truven Health Analytics MarketScan ${ }^{\circledR}$ Commercial Claims and Encounters database for the period of January 2006 to December 2012. The database includes de-identified, individual-level medical and outpatient pharmacy claims data for employees with primarily employer-based insurance and their spouses and dependents. Over 300 contributing employers and 25 nongovernment health plans in the USA contribute data to the MarketScan database, which encompassed more than 66 million individuals in the most recent year of full data collection [7]. This individual-level data allows longitudinal tracking of patients during outpatient or emergency department visits, inpatient hospital stays, and ambulatory surgeries. The MarketScan database has previously been used to evaluate delivery of care and post-operative complications after total joint arthroplasty $[16,22]$ and spine surgery $[5,6,11,14]$, as well as other types of surgical intervention. During our study period, the overall sex distribution was $49 \%$ male and $51 \%$ female. The overall age distribution was 0 to 17 years, $27.7 \%$; 18 to 30 years, $21.7 \%$; 31 to 40 years, $16 \%$; 41 to 50 years, $16.5 \%$; 51 to 60 years, $14.1 \%$; 61 to 65 years, $3.96 \%$; and over 65 years, $0 \%$.

We included patients ages 18 to 64 years of age with both an International Classification of Diseases, Ninth Revision, Clinical Modification (ICD-9-CM) diagnosis code for closed scaphoid fracture (814.01) and a Current Procedural Terminology (CPT) treatment code for scaphoid fracture treatment (splinting: 29125, 29105; casting: 29065, 29075; closed treatment: 25622 , 25624; surgery: 25628) occurring on the same date in the study cohort. Only patients with health insurance coverage for at least 6 months were eligible for inclusion, so as to allow adequate time for coding of comorbid diagnoses after health insurance coverage is established. We excluded patients with diagnosis of open scaphoid fracture (814.11), recent distal radius fracture (813.xx) or wrist ligamentous injury (carpal sprain/tear [842.00, 842.01], carpal dislocation [833.xx]) within 3 months of a closed scaphoid fracture, or any history of avascular necrosis of the scaphoid (733.09) or post-traumatic wrist arthritis (716.13, 716.14).

We identified patients who underwent treatment for scaphoid nonunion during the study period via subsequent CPT codes (repair scaphoid nonunion [25440] alone or with vascularized bone graft [25430] or free microvascular bone graft [20955]). Because we were using administrative data, we could not confirm diagnosis of nonunion. Additionally, we could not include asymptomatic nonunions or welladjusted malunions. The minimum time allowed for nonunion surgery to occur after initial treatment was 28 days; patients without one of these events by the end of the study period were censored. We did not query for any other treatments (such as continued fracture care) beyond those listed above for nonunion treatment.

We determined the incidence density of scaphoid fracture treatment per year of coverage by dividing the number of cases in the study cohort by the number of eligible insurance beneficiaries (at least 18 years of age with at least 3 months of health insurance coverage) included in the MarketScan database during the study period (2006 to 2012).

We analyzed year-to-year trends in the percentage of all scaphoid fractures that were treated surgically and the percentage of all cases that had subsequent treatment for nonunion using the Cochran-Armitage trend test.

Due to the presumed differences between patients who were treated with casting and those treated with surgery, no statistical analyses were performed to compare these groups. We compared patients who did and did not undergo treatment for a scaphoid nonunion with univariate statistical tests: Wilcoxon rank-sum tests were used for continuous variables (as these were not normally distributed) and Pearson $\chi$-square tests of independence were used for categorical variables. The presence of numerous comorbidities was tabulated from co-existing claims records based on the Charlson comorbidity index ("Electronic Supplementary Material") [15]. Some patients (1000 of the 8470 [11.8\%] patients in the "no nonunion treatment" group and 53 of the $453[11.7 \%]$ in the "underwent nonunion treatment" group) were missing comorbidity data and were excluded from analysis including the comorbidity variable. There were no significant differences in the proportion of patients with missing comorbidity data based on the initial treatment group (casting or surgery) or the presence of nonunion treatment.

Variables with $p$ values of less than 0.25 in the univariate analyses were included in a multivariate logistic regression model with scaphoid nonunion treatment as the dependent variable. Age was entered into the model as the continuous variable after dividing age into decades (age divided by 10 ; first decade ages 18 to 27 years old); this rescaling was used to facilitate interpretation of the model results. A backward stepwise method was used to remove variables until those remaining had $p$ values of 0.05 or less. After significant variables were identified, interaction effects among them were investigated.

\section{Results}

Mean age for the 453 patients undergoing nonunion treatment was significantly lower than that for those not treated for nonunion, and the former group consisted of a significantly higher proportion of male patients with a higher rate of initial surgical treatment (Table 1). The cohort was $72 \%$ male and $28 \%$ female. The most common age range was 16 
to 24 years (41\%), followed by ages 25 to 44 years (34\%) and 45 to 65 years $(25 \%)$.

From 2006 to 2012, the incidence density of scaphoid fracture treatment (both casting and surgery) was 10.6 per 100,000 person-years in the MarketScan cohort. Of the 8923 closed scaphoid fractures from 2006 to 2012, 29\% were treated surgically and $71 \%$ were treated with casting. The frequency of surgical treatment rose significantly from $22.1 \%$ in 2006 to $34.1 \%$ in 2012 ( $p<0.001$; Fig. 1). The frequency of nonunion treatment during the study period was $10.8 \%$ after surgery and $3 \%$ after casting $(p<0.001$; Fig. 1). The frequency of nonunion treatment did not change over time for either the surgery or casting groups (or for the overall cohort). The average age of patients with a scaphoid fracture nonunion treatment (28.2 years) was lower than those without treatment for nonunion (33.9 years) $(p<0.001)$. Univariate statistical analysis results for data on demographics, original treatment type, and comorbidity are shown in Table 1.

Patients who underwent treatment for scaphoid nonunion were significantly more likely to be male (OR, 1.89; 95\% CI, 1.4-2.56) or have undergone prior surgical treatment (OR, $3.03 ; 95 \%$ CI, 2.5-3.7). The youngest patients are at significantly higher odds of being treated for a nonunion, with the risk of treatment for nonunion decreasing by $18 \%$ with each decade of age beyond 18 to 27 years (OR, 0.82; 95\% CI, 0.75-0.89). Covariates in the final multivariate model are sex, surgical treatment, decade of age, and a small but significant $(p=0.04)$ interaction effect of decade of age and surgical treatment. This interaction effect does not change the statistical significance and interpretation of the main effects and was excluded from the final model for simplicity. The number of comorbidities was not a statistically significant variable in the final regression model.

\section{Discussion}

There currently is a lack of clarity regarding the incidence of and epidemiologic burden related to scaphoid fractures in the general population. A population-based assessment of the trends in scaphoid fracture treatment and rates of union would be helpful to better understand the relative value of each treatment option. The goal of this study was to estimate the incidence density of scaphoid fractures, the frequency of subsequent treatment for nonunion, and risk factors for nonunion treatment. An additional goal was to analyze trends in the treatment for scaphoid fractures and the frequency of scaphoid nonunion treatment from 2006 to 2012. Our primary hypothesis was that the incidence density of scaphoid fractures would be higher than previously estimated and our secondary hypothesis that the frequency of surgical treatment would increase over time using trend analysis. To summarize our results, we estimated the incidence of scaphoid fractures as 10.6 per 100,000 personyears. Of 8923 closed scaphoid fractures, 29 and $71 \%$ were treated surgically and with casting, respectively. The frequency of surgical treatment significantly rose from $22.1 \%$ in 2006 to $34.1 \%$ in 2012 . The frequency of nonunion treatment was $10.8 \%$ after surgery and $3 \%$ after casting; neither changed over time. Younger age, male sex, and surgical treatment are associated with a higher risk of nonunion treatment.

The current study carries limitations inherent to the use of administrative data, such as a reliance on accurate coding. Most notably, we arbitrarily designated subsequent treatments at least 28 days from initial treatment as treatments for nonunion. This may lead to the categorization of some cases, such as delayed decisions for surgery or discovery of fracture displacement after original coding as non-operative

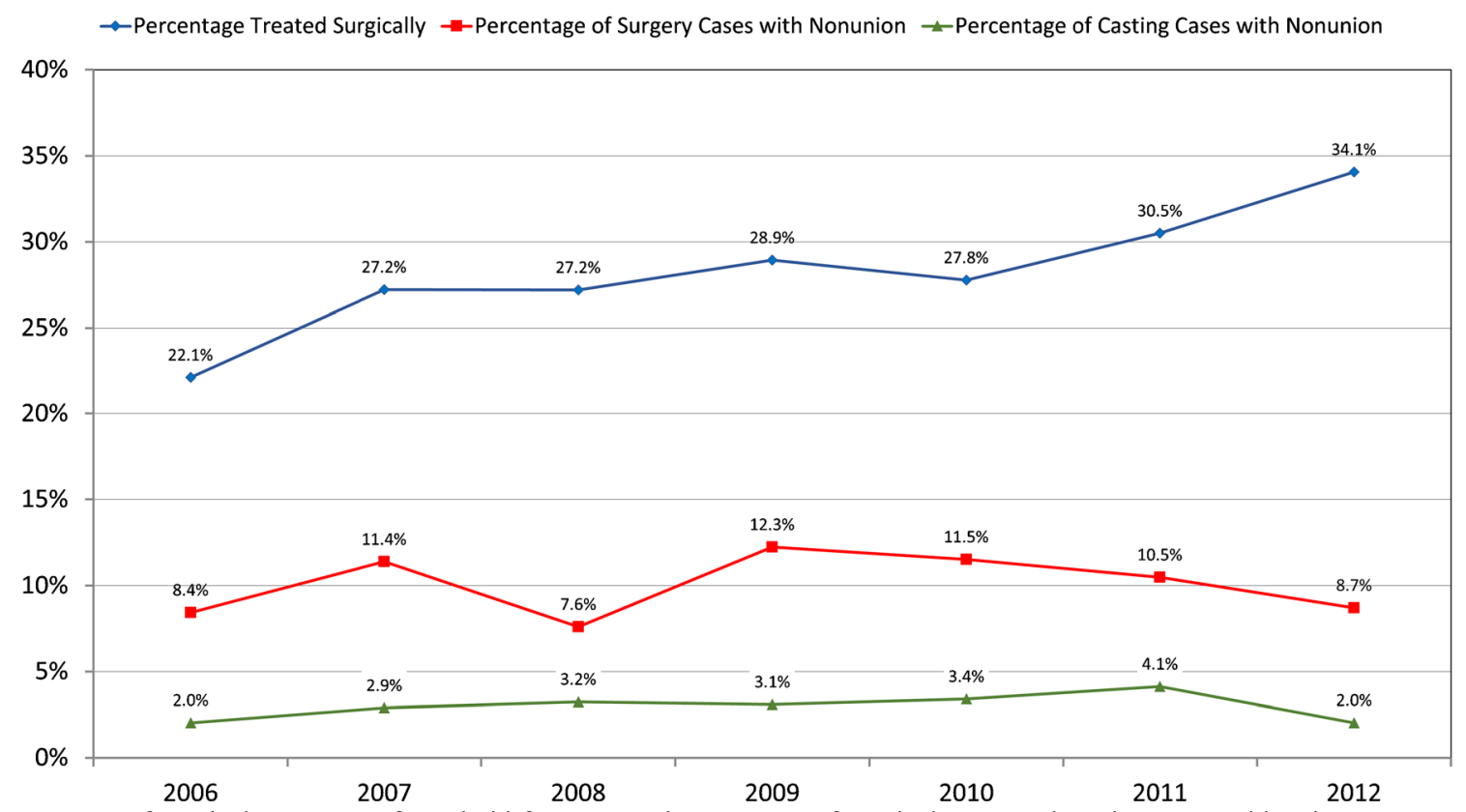

Fig. 1. Frequency of surgical treatment of scaphoid fractures and percentage of surgical cases and casting cases with subsequent treatment for nonunion over a period of 6 years. 
Table 1 Summary of demographic, original treatment type, and comorbidity data

\begin{tabular}{lll}
\hline & $\begin{array}{l}\text { No nonunion treatment } \\
(n=8470)\end{array}$ & $\begin{array}{l}\text { Underwent nonunion treatment } \\
(n=453)\end{array}$ \\
\hline Age & 33.9 years (SD 14.3 years) & $\begin{array}{l}28.2 \text { years (SD 11.9 years) } \\
p<0.001 \text { (Wilcoxon rank-sum) }\end{array}$ \\
Sex & & $396(87 \%)$ \\
Male & $5938(70 \%)$ & $57(13 \%)$ \\
Female & $2532(30 \%)$ & $p<0.001(\chi$-square $)$ \\
Type of original treatment & & $192(42 \%)$ \\
Casting & $6139(72 \%)$ & $261(58 \%)$ \\
Surgical treatment & $2331(28 \%)$ & $p<0.001(\chi$-square) \\
Comorbidities* & $n=700$ \\
0 & $6550(88 \%)$ & $369(92 \%)$ \\
1 & $668(9 \%)$ & $20(5 \%)$ \\
$2+$ & $252(3 \%)$ & $11(3 \%)$ \\
\end{tabular}

* Sums of each cell for comorbidities do not match the number of patients in each group (no nonunion treatment or nonunion treatment) due to exclusion of patients who are missing comorbidity data

treatment, as treatments for nonunion. While this could bias the frequency of nonunion treatment to be higher than expected in the non-operative treatment group, our results do not reflect this. However, this timeframe allows us to capture cases that occur earlier than 6 months but are likely to be treatments for nonunion or delayed union. This is particularly useful for the cohort that was originally treated with surgery, since some surgeons may not wait as long as 6 months after surgery before intervening if a fracture has not reached union after surgery. Because we identified the study cohort by treatment codes, patients with untreated scaphoid fractures were not included in the cohort. This may lead to an underestimate of the true incidence of scaphoid fractures and the true incidence of treatment for scaphoid nonunions. We were also unable to radiographically confirm the diagnosis of scaphoid fracture in those who underwent treatment. The tendency to treat patients with suspected scaphoid fractures even for a short period may lead to a relative overestimate of the incidence of scaphoid fractures in the USA. We accept the limitations associated with the possible under- and over-estimation of scaphoid fracture incidence, as our data provide an incidence estimate that reflects practice in the USA. Also, due to the data available, we did not diagnose "nonunion" in the clinical sense; rather, we identified patients who underwent surgical treatments that are typically used to treat nonunions. While it would be ideal to diagnose the former, identifying the latter described cases that were deemed worthy of surgical intervention. We believe this provides meaningful information for the surgeon. Although these data may serve as a surrogate for practice, they directly reflect coding and billing data in the USA. Additionally, the database does not include laterality data, and we assume that initial treatment and subsequent nonunion surgeries occurred on the same side. Similarly, we were unable to determine the location of scaphoid fractures, which may limit the generalizability of our findings to specific fracture types. Additionally, we excluded patients with perilunate dislocation diagnoses but did not exclude patients with non-scaphoid carpal fractures, which introduces the possibility of including miscoded perilunate dislocations. Another limitation inherent to administrative data is the lack of detailed clinical information, which precluded us from investigating the influence of other factors (such as mechanism of injury, fracture displacement, surgeon experience, potential clustering of surgeon decision making, and patient expectations) that likely affect treatment decisions and subsequent outcomes after scaphoid fracture. This is particularly germane when considering the relationship between age and nonunion treatment, as physicians may be more inclined to offer treatment for scaphoid nonunion to younger patients than to older patients (regardless of any potential differences in the frequency of nonunion itself). The maximum length of time from initial scaphoid fracture treatment to censoring at the end of the study period is 6 years, which precludes us from evaluating the frequency of salvage procedures after scaphoid fracture treatment. Detailed demographic information that may influence outcomes, such as race, ethnicity, and household income, are not reliably coded or available in MarketScan. Additionally, because MarketScan is a proprietary database, we are unable to audit the data for coding errors. Truven Health edits procedure codes for validity; if they are invalid, they are set to missing. The MarketScan database only includes individuals with employer-based insurance in the USA, limiting the generalizability of our findings to those with employer-based commercial insurance (and not those who are uninsured or covered by Medicaid or Medicare). Furthermore, patients may lose commercial insurance coverage after their fracture has occurred but before any further treatment is given. We attempted to minimize the chance of insurance gaps by including continuous coverage of 6 months as one of the inclusion criteria. In our study, $6.2 \%$ of the included patients (with an initial fracture) lost insurance coverage before the occurrence of a future event (such as surgery for nonunion). Statistical analysis showed no difference in demographics or likelihood of future 
surgery for nonunion in those patients with loss of coverage (6.2\% of the cohort) and those who did not lose coverage. Additionally, the cross-sectional, retrospective nature of the MarketScan database limits our ability to perform direct statistical comparison between the groups treated with casting and surgery. However, analysis of the MarketScan database provides the distinct advantage of a multi-payer, "realworld" perspective across the full continuum of care delivery for patients with scaphoid fractures.

Our estimated incidence density of scaphoid fracture treatment in the USA for commercially insured patients-10.6 per 100,000 person-years from 2006 to 2012 in a commercially insured population of less than 65 years of age - is substantially higher than previously estimated for the USA by Van Tassel et al. [21]. The discrepancy is likely related to the differences in the data collection methods with regard to both timing of data capture and assessment of true versus suspected fractures. While Van Tassel et al. included only patients diagnosed with scaphoid fractures in the emergency department, our dataset captured patients with an outpatient clinical presentation that warranted treatment of a scaphoid fracture with casting or surgery. We believe that reliance on diagnoses made in the emergency department is more likely to result in an underestimation of the incidence of scaphoid fractures, as a subset of patients presenting with wrist injuries have normal radiographs and may eventually be diagnosed with scaphoid fractures after subsequent outpatient imaging. In the emergency department, less time may be spent on accurate coding. Therefore, we believe that our dataset provides a more accurate reflection of scaphoid fracture epidemiology in the USA. The sample used by Van Tassel et al. also included patients with all insurance types, including Medicare. Because scaphoid fractures are more likely to occur in younger patients, their estimated incidence may have been lower due to inclusion of older patients (and conversely, our estimated incidence density may have been higher due to exclusion of patients over age 65 years). While our estimate of scaphoid fracture incidence is lower than that of population-based reports from other countries $[9,13,15,17]$, the differences in injury epidemiology, population demographics, and health care delivery systems among countries preclude meaningful direct comparison. In a recent population-based study in Finland, trends in surgical treatment of scaphoid fractures and nonunion were evaluated [17]. The authors did not investigate the rate of non-operative scaphoid fracture treatment, which likely explains why their estimated incidence of scaphoid fracture primary fixation (5.5 to 17.8 cases per $1,000,000$ person-years) differs from the 10.6 fractures treated per 100,000 person-years observed in the current study.

A majority $(71 \%)$ of scaphoid fractures included in our study were treated non-operatively, but there was a significant increase in the overall percentage of fractures treated surgically from $2006(22.1 \%)$ to $2012(34.1 \%)$. The time period in the current study coincided with publication of multiple studies, albeit with inconsistent findings, examining the potential benefits of surgical treatment for nondisplaced and minimally displaced scaphoid fractures $[4,12,19]$. We speculate that the increased frequency of surgical treatment of scaphoid fractures seen in the current study may reflect a response by surgeons to the contemporaneous literature. Our lack of access to detailed information about patient and fracture characteristics, such as mechanism of injury and fracture displacement, limits our ability to investigate factors that may have contributed to the increasing frequency of operative management in the USA from 2006 to 2012.

The rate of nonunion treatment was $10.8 \%$ after surgical treatment and 3\% after casting and did not change over the study period in either group. This finding differs slightly from the threefold increase in scaphoid nonunion treatment observed in Finland between 1997 and 2014 by Raittio et al. [17]. The presumed differences in how patients were selected into each of these groups prohibit direct statistical comparison of the rates of nonunion treatment. Furthermore, this result should not be interpreted as a causal relationship between surgical treatment and subsequent treatment for nonunion. Injury characteristics (such as the extent of fracture displacement and the mechanism associated with injury) and patient characteristics (such as desired activity levels and expectations for functional outcomes) almost certainly contributed to both the chosen treatment and the subsequent rate of nonunion. Our reported rate of treatment for nonunion after initial surgical treatment $(10.8 \%)$ is higher than the nonunion rate reported in meta-analysis of level-I evidence $(1 \%)$, level-II evidence (3\%), and level-IV evidence (4\%) [19]. While the heterogeneity of the patients and fractures treated in our study plays a role in these discrepancies, publication bias (the general reticence to report complications or poor outcomes) may furthermore contribute to this disparity [10].

It is important to note that we are not reporting the clinical diagnosis of nonunion; rather, we are reporting those patients who underwent subsequent specific surgical treatments (coded as repair nonunion, vascularized bone grafting, or free microvascular graft) that we assume were intended to treat a nonunion. With the advantage of providing a population-based report that reflects actual practice, the rate of treatment for nonunion that we provide is well-suited for counseling patients and establishing a more appropriate benchmark to evaluate individual practice. The latter may be particularly useful for patient counseling.

In conclusion, our estimated incidence of scaphoid fracture (10.6 per 100,000 person-years) is higher than previously reported. The increased enthusiasm in the USA to surgically treat scaphoid fractures is reflected by our trend analysis. The frequency of treatment for nonunion after surgical management for closed scaphoid fractures exceeded $10 \%$ in a commercially insured population under the age of 65 . Younger patients, men, and those treated surgically are at increased risk for treatment for nonunion, all of which may reflect the higher energy mechanism of injury typically associated with scaphoid fractures. Given the increased utilization of surgery, surgeons and patients should be aware of the frequency of surgical treatment for presumed nonunion after initial surgical management to inform perioperative treatment decisions. 
Funding Information In support of this study, the authors (DAO and NHK) received a $\$ 4,995$ USD Institute of Clinical and Translational Sciences Just-In-Time Core Usage Grant from the Washington University in Saint Louis Center for Administrative Data Research.

\section{Compliance with Ethical Standards}

Conflict of Interest: Christopher J. Dy, MD, MPH; Jack Baty, MS; and Kerry Bommarito, $\mathrm{PhD}, \mathrm{MPH}$, declare that they have no conflicts of interest. Nikolas H. Kazmers, MD, MSE, and Daniel A. Osei, MD, MSc, received a \$4995 Institute of Clinical and Translational Sciences Just-InTime Core Usage Grant from the Washington University in Saint Louis Center for Administrative Data Research in support of this work.

Human/Animal Rights: All procedures followed were in accordance with the ethical standards of the responsible committee on human experimentation (institutional and national) and with the Helsinki Declaration of 1975, as revised in 2013.

Informed Consent: Informed consent was waived from all patients for being included in this study.

Required Author Forms Disclosure forms provided by the authors are available with the online version of this article.

\section{References}

1. Beeres FJ, Hogervorst M, den Hollander P, Rhemrev S. Outcome of routine bone scintigraphy in suspected scaphoid fractures. Injury. 2005;36(10):1233-1236.

2. Beeres FJ, Hogervorst M, Rhemrev SJ, den Hollander P, Jukema GN. A prospective comparison for suspected scaphoid fractures: bone scintigraphy versus clinical outcome. Injury. 2007;38(7):769-774.

3. Beeres FJ, Rhemrev SJ, den Hollander P, et al. Early magnetic resonance imaging compared with bone scintigraphy in suspected scaphoid fractures. J Bone Joint Surg Br. 2008;90(9):1205-1209.

4. Buijze GA, Doornberg JN, Ham JS, et al. Surgical compared with conservative treatment for acute nondisplaced or minimally displaced scaphoid fractures: a systematic review and metaanalysis of randomized controlled trials. J Bone Joint Surg Am. 2010;92(6):1534-1544.

5. Cahill KS, McCormick PC, Levi AD. A comprehensive assessment of the risk of bone morphogenetic protein use in spinal fusion surgery and postoperative cancer diagnosis. J Neurosurg Spine. 2015;10:1-8.

6. Cole T, Veeravagu A, Zhang M, Ratliff JK. Surgeon procedure volume and complication rates in anterior cervical discectomy and fusions: analysis of a national longitudinal database. J Spinal Disord Tech. 2014; 30(5):E633-E639.

7. Danielson E. White paper: health research data for the real world: the MarketScan® databases. Truven Health Analytics, Inc.; 2014. http://truvenhealth.com/Portals/0/Users/031/31/31/ PH 13434\%200314_MarketScan_WP web.pdf.

8. Dias JJ, Brenkel IJ, Finlay DB. Patterns of union in fractures of the waist of the scaphoid. J Bone Joint Surg Br. 1989;71(2):307310.

9. Duckworth AD, Jenkins PJ, Aitken SA, et al. Scaphoid fracture epidemiology. J Trauma Acute Care Surg. 2012;72(2):E41-45.

10. Hasenboehler EA, Choudhry IK, Newman JT, et al. Bias towards publishing positive results in orthopedic and general surgery: a patient safety issue? Patient Saf Surg. 2007;1(1):4.

11. Huang KT, Hazzard M, Thomas S, et al. Differences in the outcomes of anterior versus posterior interbody fusion surgery of the lumbar spine: a propensity score-controlled cohort analysis of 10,941 patients. J Clin Neurosci. 2015;22(5):848-853.

12. Ibrahim T, Qureshi A, Sutton AJ, Dias JJ. Surgical versus nonsurgical treatment of acute minimally displaced and undisplaced scaphoid waist fractures: pairwise and network meta-analyses of randomized controlled trials. J Hand Surg Am. 2011;36(11):1759-1768 e1751.

13. Jonsson BY, Siggeirsdottir K, Mogensen B, Sigvaldason H, Sigursson G. Fracture rate in a population-based sample of men in Reykjavik. Acta Orthop Scand. 2004;75(2):195-200.

14. Kastenberg ZJ, Hurley MP, Weiser TG, et al. Adding insult to injury: discontinuous insurance following spine trauma. $J$ Bone Joint Surg Am. 2015;97(2):141-146.

15. Larsen CF, Brondum V, Skov O. Epidemiology of scaphoid fractures in Odense, Denmark. Acta Orthop Scand. 1992;63(2):216-218.

16. Miletic KG, Taylor TN, Martin ET, Vaidya R, Kaye KS. Readmissions after diagnosis of surgical site infection following knee and hip arthroplasty. Infect Control Hosp Epidemiol. 2014;35(2):152-157.

17. Raittio LT, Jokihaara J, Huttunen TT, et al. Rising incidence of scaphoid fracture surgery in Finland. J Hand Surg Eur Vol. 2018:43(4):402-406. https://doi.org/10.1177/1753193417726051

18. Schuind F, Haentjens P, Van Innis F, et al. Prognostic factors in the treatment of carpal scaphoid nonunions. J Hand Surg Am. 1999;24(4):761-776.

19. Suh N, Benson EC, Faber KJ, Macdermid J, Grewal R. Treatment of acute scaphoid fractures: a systematic review and meta-analysis. Hand (NY). 2010;5(4):345-353.

20. Trumble TE, Vo D. Proximal pole scaphoid fractures and nonunion. J Hand Surg Am. 2001;1(3):155-171.

21. Van Tassel DC, Owens BD, Wolf JM. Incidence estimates and demographics of scaphoid fracture in the US population. $J$ Hand Surg Am. 2010;35(8):1242-1245.

22. Yacub JN, Rice JB, Dillingham TR. Nerve injury in patients after hip and knee arthroplasties and knee arthroscopy. Am J Phys Med Rehabil. 2009;88(8):635-641. 